\title{
Neural Activity Relating to Generation and Representation of Galvanic Skin Conductance Responses: A Functional Magnetic Resonance Imaging Study
}

\author{
Hugo D. Critchley, ${ }^{1,2}$ Rebecca Elliott, ${ }^{3}$ Christopher J. Mathias, ${ }^{2,4}$ and Raymond J. Dolan ${ }^{1}$ \\ ${ }^{1}$ Wellcome Department of Cognitive Neurology, London WC1N 3BG, United Kingdom, ${ }^{2}$ Autonomic Unit, National Hospital \\ for Neurology and Neurosurgery, London WC1N 3BG, United Kingdom, ${ }^{3}$ Department of Psychiatry, University of \\ Manchester, Manchester M13 9PT, United Kingdom, and 4Department of Neurovascular Medicine, St. Mary's Hospital, \\ Paddington, London W2 1NY, United Kingdom
}

Central feedback of peripheral states of arousal influences motivational behavior and decision making. The sympathetic skin conductance response (SCR) is one index of autonomic arousal. The precise functional neuroanatomy underlying generation and representation of SCR during motivational behavior is undetermined, although it is impaired by discrete brain lesions to ventromedial prefrontal cortex, anterior cingulate, and parietal lobe. We used functional magnetic resonance imaging to study brain activity associated with spontaneous fluctuations in amplitude of SCR, and activity corresponding to generation and afferent representation of discrete SCR events. Regions that covaried with increased SCR included right orbitofrontal cortex, right anterior insula, left lingual gyrus, right fusiform gyrus, and left cerebellum. At a less stringent level of significance, predicted areas in bilateral medial prefrontal cortex and right inferior parietal lobule covaried with SCR. Generation of discrete SCR events was associated with significant activity in left medial prefrontal cortex, bilateral extrastriate visual cortices, and cerebellum. Activity in right medial prefrontal cortex related to afferent representation of SCR events. Activity in bilateral medial prefrontal lobe, right orbitofrontal cortex, and bilateral extrastriate visual cortices was common to both generation and afferent representation of discrete SCR events identified in a conjunction analysis. Our results suggest that areas implicated in emotion and attention are differentially involved in generation and representation of peripheral SCR responses. We propose that this functional arrangement enables integration of adaptive bodily responses with ongoing emotional and attentional states of the organism.

Key words: arousal; attention; decision-making; electrodermal; event-related fMRl; functional anatomy; galvanic skin response; neuroimaging; orbitofrontal cortex; skin conductance; somatic marker hypothesis; sudomotor; sympathetic nervous system; ventromedial prefrontal cortex
Cognitive, emotional, and physical behaviors involve changes in peripheral autonomic activity. Skin conductance (electrodermal activity) measures reflect sympathetic tone and is frequently used as an indirect measure of attention, cognitive effort, or emotional arousal. Brain mechanisms underlying generation of skin conductance response (SCR) are also integrated with those involved in emotional processing (Buchel et al., 1998). However, little is known concerning the functional neuroanatomy of central regulation and representation of SCR.

Insights into the neuronal basis of SCR have come from brain lesion studies, electrical stimulation, and functional imaging. Impaired SCR is reported in patients with discrete brain lesions of right hemisphere (Oscar-Berman and Gade, 1979; Zoccolotti et al., 1982) and of bilateral ventromedial prefrontal cortex, bilateral anterior cingulate gyrus, right inferior parietal lobe (Tranel and Damasio, 1994), and amygdala (Bechara et al., 1995, 1999). Lesions to amygdala, a region anatomically and functionally interconnected with ventromedial prefrontal cortex, also impair SCR

\footnotetext{
Received Dec. 6, 1999; revised Jan. 21, 2000; accepted Jan. 24, 2000.

R.J.D. is funded by a Program Grant from the Wellcome Trust; H.D.C. is funded by a Project Grant from the Brain Research Trust. The help and advice of O. Josephs and Drs. D. Corfield, J. Armony, R. Henson, and C. Büchel are gratefully acknowledged.

Correspondence should be addressed to Dr. Hugo D. Critchley, Wellcome Department of Cognitive Neurology, Institute of Neurology, 12 Queen Square, London WC1N 3BG, United Kingdom. E-mail: hugo@fil.ion.ucl.ac.uk.

Copyright (C) 2000 Society for Neuroscience $0270-6474 / 00 / 203033-08 \$ 15.00 / 0$
}

during aversive conditioning and reward-related feedback $(\mathrm{Be}-$ chara et al., 1999), but not in response to unconditioned aversive stimuli (Tranel and Damasio, 1989). Ventromedial prefrontal lesions, like amygdala lesions, impair anticipatory SCR during risk-related decision making, but unlike amygdala lesions they do not impair SCR elicited by reward-related feedback (Bechara et al., 1999). Modulation of SCR can also be elicited by electrical stimulation of amygdala, hippocampus, anterior cingulate, and frontal cortex (Mangina and Beuzeron-Mangina, 1996). Positive correlations between SCR and neural activity in motor cortex and mid-cingulate in subjects experiencing emotive stimuli are reported in functional imaging studies (Fredrikson et al., 1998). In an event-related functional magnetic resonance imaging (fMRI) study, activity in amygdala and insula reflected acquisition of aversive conditioning, indexed by SCR (Buchel et al., 1998).

The importance of afferent feedback of arousal includes an influence on emotion and cognition. James (1894) proposed that autonomic activity is the essence of emotion and that subjective "feelings" are merely an interpretation of perceived visceral responses. More recently, Damasio's “somatic marker hypothesis" suggests that the feedback of arousal, in addition to generating feeling states, may bias social behavior and decision making (Damasio et al., 1991; Damasio, 1994). Arousal mechanisms are also reported as influencing encoding of memories, particularly emotional memories (Cahill, 1997). Despite a wealth of neuropsychological data, the functional neuroanatomy underlying the 
representation of somatic arousal (and skin conductance in particular) during decision making associated with risk and reinforcement has not been systematically examined in normal subjects. Thus, we used fMRI to identify brain areas involved in somatic arousal and to differentiate activity relating to generation and afferent representation of skin conductance response.

\section{MATERIALS AND METHODS}

Subjects. We recruited six right-handed healthy volunteers (mean age 34 years \pm 7 ; three male and three female) who gave full, informed consent to participate in a research study approved by the National Hospital for Neurology and Neurosurgery and the Institute of Neurology Joint Medical Ethics Committee. Before scanning, subjects were pretrained on a decision-making task in which they were required to select one of two visually presented playing cards, before visual feedback informed them whether their selection was correct or incorrect, resulting in monetary gain or loss (see below). Subjects were naive to the fact that their response selections did not influence the feedback provided.

Experimental paradigm. To examine sympathetic arousal (indexed by $\mathrm{SCR}$ ) in the context of risk-taking behavior, subjects were scanned while performing a decision-making task with immediate reinforcement (reward and punishment). Subjects were presented a series of pairs of stimuli depicting playing cards, one red and one black, and were told that on half the trials the red card was correct and on the other half the black card was correct. The task was to guess the correct card on each trial and respond with a button press (right index finger). A tick and a cross denoted, respectively, whether a response had been correct or incorrect on any particular trial. At the side of the presentation screen a bar displayed a cumulative "reward" score across all the trials. The height of this bar increased by one increment for every correct response and decreased by one increment, representing $£ 1$, for every incorrect response (Fig. 1A). Each trial lasted $3.5 \mathrm{sec}$, and there was a total of 288 trials equivalent to $16.8 \mathrm{~min}$. Although subjects attempted to determine strategies to maximize their winnings (confirmed at debriefing after scanning), their responses in fact did not influence the feedback they received, which was predetermined by a constrained binomial random walk function and was the same for each subject (Fig. $2 A$ ).

fMRI and skin conductance data acquisition. Subjects were scanned during task performance using a Siemens VISION system at 2 Tesla to acquire gradient-echo, echoplanar T2*-weighted images with BOLD (blood oxygenation level dependent) contrast. Each volume comprised $48 \times 3 \mathrm{~mm}$ axial scans with $3 \mathrm{~mm}$ inplane resolution, and volumes were continuously acquired every $4.2 \mathrm{sec}$. Each run began with six "dummy" volumes to allow for T1 equilibration effects, which were subsequently discarded. Additionally, a T1-weighted structural image was acquired in each subject for registration with the functional BOLD data. Comparison of the raw functional data with this structural scan also enabled us to estimate for each subject the extent of signal dropout attributable to the susceptibility artifact. During task performance, skin conductance was continuously monitored using silver electrodes taped to the palmar surface of the left index and middle fingers, and the signal was fed via a skin conductance processing unit (SCL 200, Biofeedback Systems, Manchester, UK). The filtered analog output of the SCR was displayed online and recorded digitally (sample rate, $100 \mathrm{~Hz}$ ) using SPIKE 2 software (CED, Cambridge, UK). During echo planar imaging data acquisition, the skin conductance trace was contaminated by highfrequency noise that was common to each volume acquisition. Off-line post-processing of the digitalized skin conductance data enabled the removal of this repetitive signal contamination, and further filtering and smoothing were used to remove nonrepetitive noise. The skin conductance traces were then resampled at the frequency of volume acquisition.

Data analysis. We performed two types of analysis on these data to determine the central representation of SCR. First, we examined regional brain activity covarying with SCR over the scanning period (covariance analysis). Second, we used an event-related analysis of the SCR (achieved by modeling peaks of SCR activity as $\delta$ functions) to determine the temporal relationship of neural activity to discrete SCR events. Data were analyzed using Statistical Parametric Mapping (SPM99, Wellcome Department of Cognitive Neurology, London, UK) where statistical inferences were based on the theory of random Gaussian fields (Friston et al., 1995a-c). Descriptions of the anatomical localization were determined using averaged structural MRIs and the atlas of Duvernoy (1991).

Covariance analysis. Three statistical regressors were derived from the
A
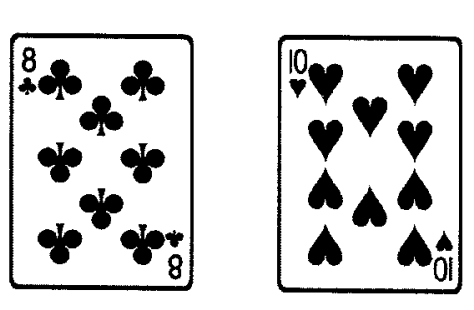

?

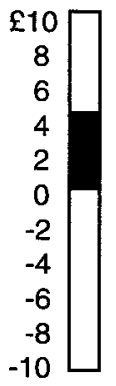

B
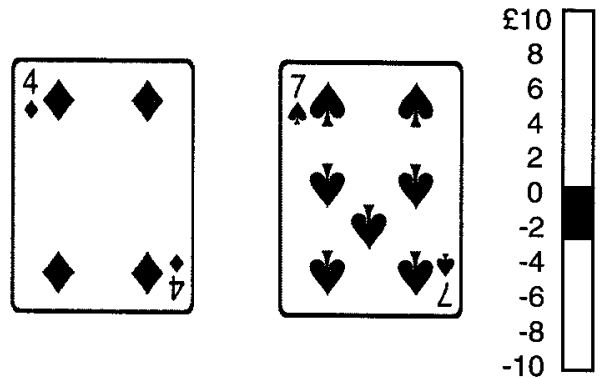

?

Figure 1. Experimental task paradigm. For each trial, pairs of cards were presented on one side of the screen with the cumulative reward bar on the other. Subjects were required to respond to one of the pair of cards after which feedback was given (tick or cross), and the reward bar was adjusted accordingly to indicate monetary gain or loss. The figure illustrates $(A)$ decision making during cumulative gain and $(B)$ decision making during cumulative loss.

filtered SCR trace. For each subject, SCR was convolved with a canonical hemodynamic response function (HRF) to provide a physiological offset to optimize identification of brain regions representing the feedback of peripheral SCR. This provided the principal regressor-of-interest. The other regressors were the first and second order temporal derivatives of the HRF-convolved SCR. These were calculated to account for short temporal differences between the principal regressor-of-interest and associated regional changes in BOLD signal. A design matrix was constructed to provide the statistical model, into which the three regressors derived from the SCR recording were entered. To disambiguate SCRrelated activity from activity directly induced by task-related processing of stimulus and reinforcement, we included three regressors that represented height of cumulative reward bar, i.e., overall winnings or losses (regressor convolved with a canonical HRF function), changes in winnings (temporal derivative of reward bar regressor), and the interaction between the height of bar and the change in height of the bar (representing losses after winning streaks and wins after losing streaks, etc.). The first 20 scans acquired during the task performance and the first 20 values of the regressors were not entered into the analysis to eliminate the nonspecific skin conductance orienting response that occurred in all subjects at the onset of the task performance. Thus, for each subject, fMRI data from 220 scans and corresponding values for the regressors (reflecting brief spontaneous fluctuations in SCR during task performance and their first and second order temporal derivatives) were entered into the design matrix.

Global signal changes were removed using proportional scaling. In the data analysis, we modeled the evoked regional signal changes using the principal regressor-of-interest. Thus, areas positively covarying with this regressor corresponded to activity consequent to increased arousal, indexed by increases in SCR. Effects at each and every $\left(2 \mathrm{~mm}^{3}\right)$ voxel were estimated, and regionally specific effects were compared using linear 
contrasts in a fixed effects model. The total search volume was 207,072 voxels. The resulting set of voxels constituted a statistical parametric map (SPM) of the $t$ statistic [SPM(t)], which was then transformed into the unit distribution, SPM $(\mathrm{Z})$. To deconfound SCR-related activity from activity resulting directly from task performance, a statistical parametric map of the $F$ statistic [SPM $(F)]$ of the three task-related regressors was used as an exclusive mask when reporting regional activity covarying with SCR. The threshold for this mask was set at $p<0.0001$, the same level at which we report uncorrected $p$ values for significant activation.

Event-related analysis. In the event-related analysis of the SCR, we identified peaks of SCR activity from a subject's SCR trace greater than twice the SD of background activity and having typical waveforms of an evoked SCR event. The maxima of these peaks were modeled with $\delta$ (stick) functions, with the height of the peaks as modulatory parameters within the model. Activity occurring in the first 20 scans of the task were again excluded. An average of 14 such events were included for each subject over the whole experiment. The time course for generation and feedback of SCR responses [informed by our own observations and those of previous studies (Buchel et al., 1998)] was estimated at between 4 and $5 \mathrm{sec}$. To determine the temporal relationship with brain activity, two vectors of onsets for the SCR events were included in the design matrix. First, we assumed that activity preceding the SCR events by $4.2 \mathrm{sec}$ reflected generation of the SCR, whereas we assumed that activity after SCR event (by $4.2 \mathrm{sec}$ ) reflected afferent feedback of peripheral states of arousal. Events were modeled with a canonical HRF. Also included in the design matrix were the three task-related regressors. Global signal changes were removed using proportional scaling. In the analyses, we examined activity synchronous with the SCR event and activity preceding (early) and following (late) the SCR event. The contrast of early and late SCR events were orthogonal with respect to each other and other regressors in the design matrix, enabling the dissociation of activity corresponding to representation and generation of electrodermal responses. To further exclude activity directly related to the reward components of the tasks, we used the F-contrast of the three task-related regressors as an exclusive mask in the analyses.

\section{RESULTS}

\section{Task performance}

Subjects were debriefed after performance of the task. All subjects reported attempting to solve the task by generating hypotheses about the relationship of feedback to the order, value, position, and color of the cards and were surprised or disappointed to learn that the feedback was unrelated to their efforts.

\section{Skin conductance responses}

All subjects exhibited a nonspecific orienting response at the onset of the task, which was subsequently excluded from the analysis. In the remainder of the task, spontaneous deviations from a steady baseline SCR were observed, which had a characteristic waveform typical of sympathetic electrodermal activity. These responses varied across subjects in their frequency and temporal relationship to the task, consistent with individualized cognitive strategies and sensitivity to reinforcement feedback that probably generated these SCR responses (Fig. 2).

\section{Brain activity associated with SCR: covariance analysis}

To identify brain regions in which activity reflected the occurrence of increased sympathetic arousal across the group, we performed a voxel-wide analysis of signal changes covarying positively with increased amplitude of regressor derived from the SCR. To ensure that our results were not confounded by the direct effects of task performance, we masked this analysis using an exclusive mask derived from the F-contrast $(p<0.0001)$ of task-related regressors that were also entered into the design matrix.

Significant ( $p<0.05$, corrected at voxel and cluster level) SCR-related activity was observed in right lateral orbitofrontal
A) Binomial random walk function

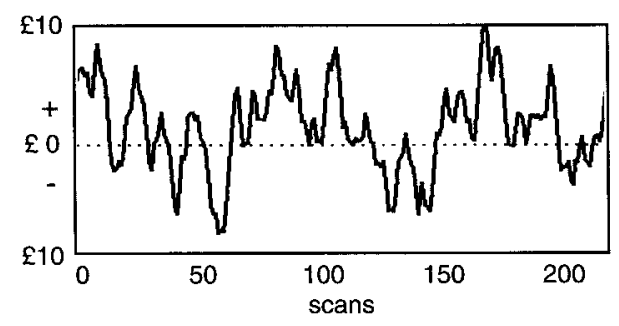

B) Skin conductance responses
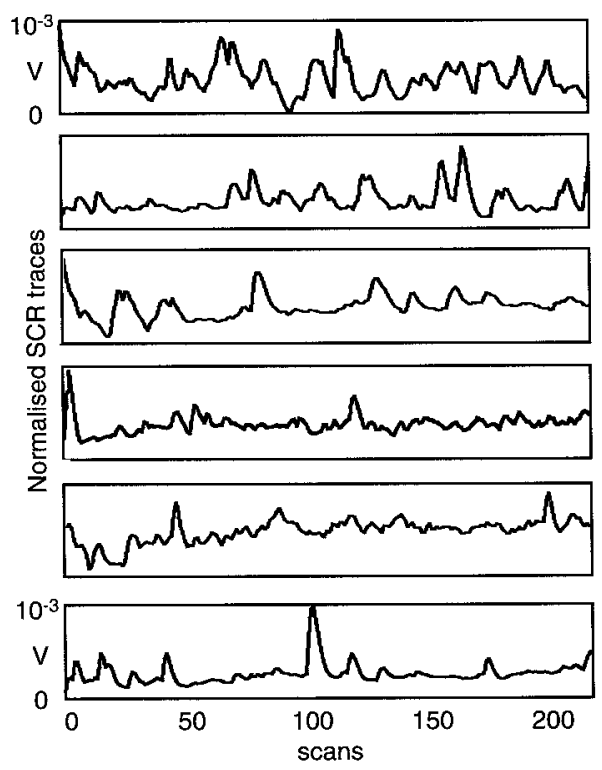

C) Example of modelling of SCR events

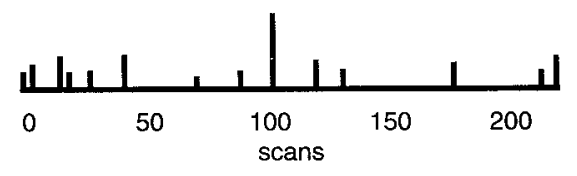

Figure 2. Binomial random walk function and skin conductance changes for each subject. $A$, The sequence of outcomes derived from a constrained binomial random walk function. This same function determined feedback outcomes in all subjects who consequently experienced the same sequence of reward and punishment. $B$, Normalized skin conductance responses for each subject over the course of the experiment from which the subjectspecific regressors-of-interest were derived. $C$, Example of modeling of SCR events with $\delta$ functions parametrically modulated by height of SCR peaks.

cortex, extending posteriorly into anterior insula, and in left lingual gyrus, right fusiform gyrus, and left cerebellum. At a less stringent level of significance ( $p<0.0001$, uncorrected), areas of SCR-related activity were evident in bilateral medial prefrontal lobe [Brodmann's area (BA) 10] and right inferior parietal lobule (Table 1, Figs. 3, 4). Brain areas in which activity decreased with increasing SCR were identified using the negative contrast of the regressor-of-interest, masked with the F-contrast of the taskrelated regressors. A significant negative relationship between SCR and regional activity was observed in premotor, supplementary motor, and posterior parietal regions ( $p<0.0001$, uncorrected) (Table 1). 


\begin{tabular}{|c|c|c|c|c|c|}
\hline Brain region & Brodmann area & Side & Number of voxels & $Z$-score & Talairach coordinates ${ }^{a}$ \\
\hline \multicolumn{6}{|c|}{ Activity positively covarying with SCR } \\
\hline Cerebellum & & $\mathrm{L}$ & 250 & $5.42^{b, c}$ & $-32-50-40$ \\
\hline Lingual gyrus & 18 & $\mathrm{~L}$ & 123 & $4.97^{b, c}$ & $-14-84-2$ \\
\hline Orbitofrontal cortex & $11 / 47$ & $\mathrm{R}$ & 270 & $4.88^{b, c}$ & $3626-12$ \\
\hline Fusiform gyrus & 19 & $\mathrm{R}$ & 258 & $4.73^{b, c}$ & $40-804$ \\
\hline Inferior parietal lobule & $41 / 40$ & $\mathrm{R}$ & 61 & 4.21 & $42-3424$ \\
\hline Medial prefrontal cortex & 10 & $\mathrm{R}$ & 45 & 4.11 & $1452-6$ \\
\hline Medial prefrontal cortex & 10 & $\mathrm{~L}$ & 61 & 4.07 & -8526 \\
\hline \multicolumn{6}{|c|}{ Activity negatively covarying with SCR } \\
\hline Premotor cortex & 6 & $\mathrm{~L}$ & 722 & $5.47^{b, c}$ & -42449 \\
\hline Supplementary motor area & 6 & $\mathrm{~L}$ & 82 & $4.66^{b, c}$ & -2854 \\
\hline Superior parietal lobule & 7 & $\mathrm{~L}$ & 87 & 4.41 & $-36-6256$ \\
\hline Supplementary motor area & 6 & & 38 & 4.21 & $0-858$ \\
\hline Supplementary motor area & 6 & & 25 & 4.19 & 01246 \\
\hline Precuneus & 7 & & 26 & 3.92 & $0-7254$ \\
\hline
\end{tabular}

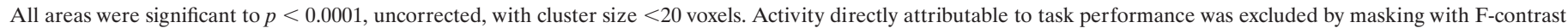
voxels of the three task-related regressors at $p<0.0001$. L, Left; R, right.

${ }^{a}$ Coordinates are given for the stereotactic space of Talairach and Tournoux (1988).

${ }^{b}$ Voxel-level significance at $p<0.05$ corrected for whole brain.

${ }^{c}$ Cluster-level significance at $p<0.05$ corrected for whole brain.

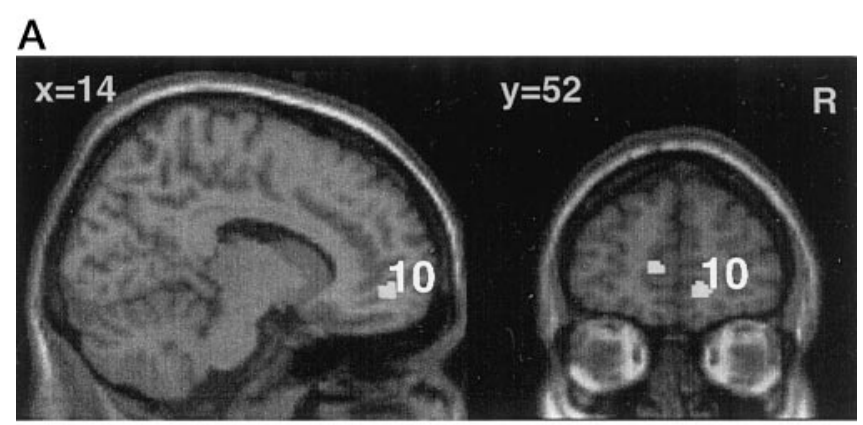

B

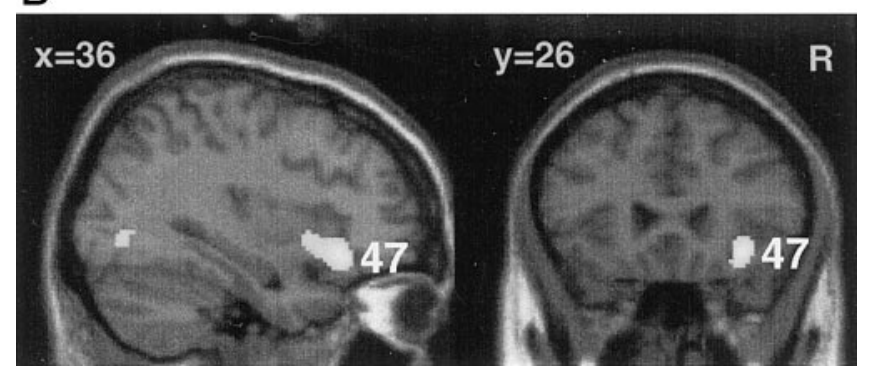

Figure 3. Anterior cerebral activity covarying with skin conductance response. $A$, Medial prefrontal activity (BA10). $B$, Right orbitofrontal activity (BA47) extending into the anterior insula. $x$, Talairach coordinate in millimeters lateral to midline; $y$, Talairach coordinate in millimeters anterior to anterior commissure; 10, Brodmann's area 10; 47, Brodmann's area $47 ; R$, right. Areas of significant activity ( $p<0.0001$, uncorrected) are mapped onto a template structural MRI scan derived from one subject.

\section{Brain activity associated with discrete SCR-events: event-related analyses}

The previous analysis cannot distinguish between activity associated with generation or feedback representation of SCR. To dissociate activity related to generation and representation of electrodermal responses, we modeled peaks in the SCR trace as
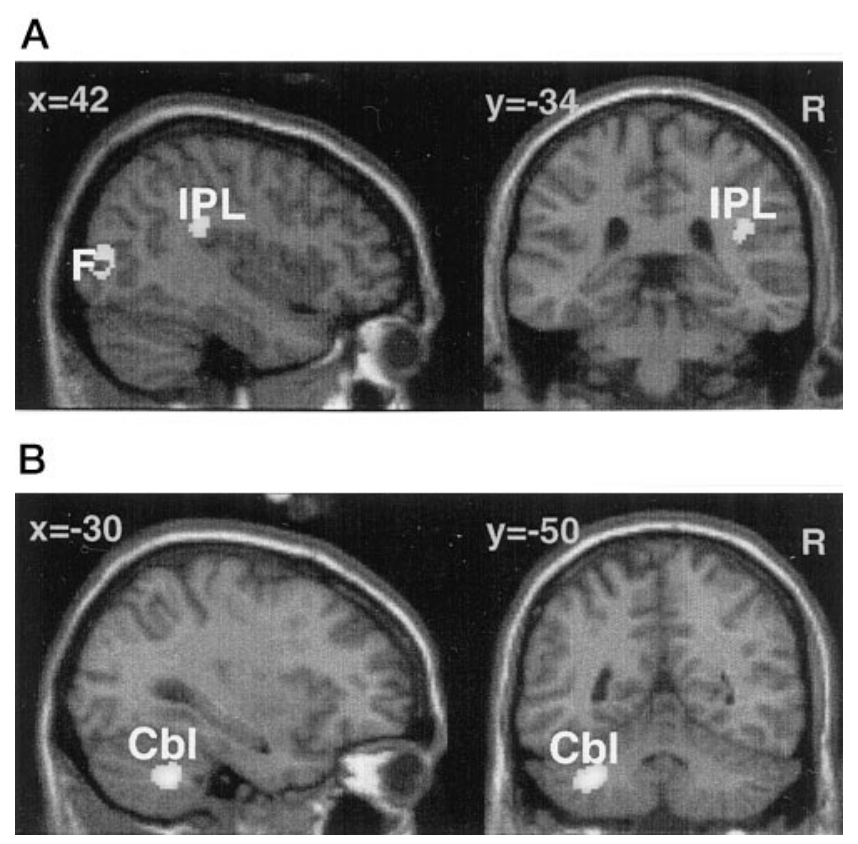

Figure 4. Parietal and cerebellar activity covarying significantly with skin conductance response. $A$, Right parietal activity (and activity in right fusiform gyrus). $B$, Left cerebellar activity. $x$, Talairach coordinate in millimeters lateral to midline; $y$, Talairach coordinate in millimeters anterior to anterior commissure; IPL, inferior parietal lobule; $F$, fusiform gyrus; $C b l$, cerebellum; $R$, right. Areas of significant activity $(p<0.0001$, uncorrected) are mapped onto a template structural MRI scan derived from one subject.

events and examined regional activity preceding and subsequent to these events (Figs. 2C, 5). Significant event-related activity preceding peaks in SCR was observed bilaterally in cerebellum (left cerebellum, $p<0.05$, corrected) and extrastriate visual cortices, and in left medial prefrontal lobe (area 10) $(p<0.0001)$ (Table 2, Fig. $6 B$ ). In the contrast for late SCR events, significant $(p<0.05$, corrected) activation was noted in right medial pre- 


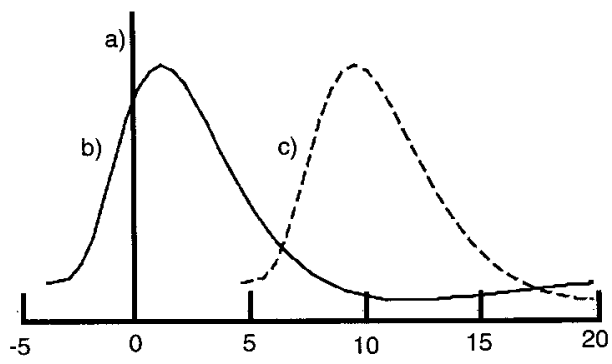

Time (seconds) from discrete skin-conductance events

Figure 5. Time line for event-related analyses. $a$, SCR event (peaks in SCR trace); $b$, response function used to model hemodynamic changes consequent to activity $4.2 \mathrm{sec}$ before SCR event (i.e., reflecting generation of SCR event); and $c$, response function used to model hemodynamic changes consequent to activity $4.2 \mathrm{sec}$ after SCR event (i.e., reflecting afferent feedback of arousal).

frontal cortex associated with representation of peripheral states of arousal (SCR) (Table 2, Figs. 6C, 7).

On the basis of neurophysiological studies reporting colocalization of neurons involved in generating and representing autonomic responses (Cechetto and Saper, 1987), we investigated which brain regions demonstrated significant responses both preceding and subsequent to SCR events (consistent with a role in generating and representing SCR). We again observed significant common activity in left medial prefrontal lobe and lingual gyrus ( $p<0.05$, corrected) and, at a less stringent level of significance, in right posterior cingulate, medial prefrontal cortex, orbitofrontal cortex, extrastriate cortex, and cerebellum ( $p<0.0001$, uncorrected) (Fig. 6D).

Assessment of raw functional data for each subject indicated a characteristic distribution of signal dropout, attributable to the susceptibility artifact. This affected the most rostral extent of frontal pole and caudal regions of ventromedial prefrontal cortex. Thus, the significant activations we observed in medial prefrontal cortices may have extended into medial orbitofrontal cortex.

\section{DISCUSSION}

In this study, we used fMRI to investigate neural activity related to generation and afferent representation of somatic arousal. Thus, in the covariance analysis, SCR measurements were convolved with a canonical hemodynamic response function, modeling temporal delay of BOLD signal changes after electrodermal responses. However, using an event-related approach we were able to examine activity occurring both before and after SCR events (discrete peaks in SCR). The fluctuations in SCR were elicited during performance of a decision-making task with reinforcement and most likely represent individualized changes in arousal and emotional tone related to anticipatory, reward, and punishment components of the task. However, in all of these analyses, we applied a mask to the analysis of SCR-related brain activity to exclude areas in which activity was directly related to reward components of the task (i.e., cumulative gain, wins and losses, and the interaction between feedback and current winnings).

We observed significant covariance between the activity of discrete cortical brain areas and sympathetic skin conductance response, an autonomic reflex that is used as an index of attention, cognitive effort, and emotion. Using an event-related approach, we were able to dissociate brain areas involved in generation and afferent representation of SCR as well as identify areas common to generation and representation of SCR events. Our findings provide evidence for involvement of areas implicated in emotional behavior and attention, in mechanisms of arousal, and particularly implicate ventromedial prefrontal regions. These findings are consistent with a proposal that peripheral autonomic states are centrally integrated with systems important for motivational behavior.

Cerebral activity covaried with SCR in orbitofrontal and medial prefrontal cortex, anterior insula, extrastriate visual cortices, parietal lobe, and cerebellum. It is noteworthy that SCR-related activity was predominantly lateralized to the right hemisphere. The most extensive cluster was located in right orbitofrontal cortex (BA 47) and extended medially into orbitofrontal area 11 and posterior into right anterior insula. Electrophysiological studies in monkeys report neurons within this orbitofrontal region that are involved in flexible representation of reinforcement (Rolls et al., 1996) and are modulated by changes in peripheral somatic state, e.g., satiety (Critchley and Rolls, 1996). This orbitofrontal area is lateral to the ventromedial prefrontal region implicated by lesion studies in generation of electrodermal responses (Tranel and Damasio, 1994). However, right-sided orbitofrontal/insula involvement is consistent with evidence reporting cardiovascular sympathetic arousal after right insula stimulation (Oppenheimer et al., 1992) and with neuroimaging studies in which cardiovascular arousal is correlated with activity in right anterior cingulate and right insula (Critchley et al., 2000). Significant right orbitofrontal activity was also observed in the eventrelated conjunction analysis, suggesting that this region is involved in both generation and afferent feedback representation of arousal. However, there was less activation of this area in the event-related than the covariance analysis, suggesting perhaps that lateral orbitofrontal cortex is tuned more to overall sympathetic tone than to rapid fluctuations in arousal that correspond to SCR events.

Lesion studies have strongly implicated ventromedial prefrontal cortex in both generation and feedback representation of arousal (Damasio et al., 1991; Tranel and Damasio, 1994; Bechara et al., 1999). In the covariance analysis, we observed SCR-related activity within bilateral medial prefrontal cortex (area 10), a region subsumed by the ventromedial prefrontal cortex area that is most consistently associated with defective SCR in patients with lesions. This region is also implicated in the integration of arousal with social and emotional behavior (Damasio et al., 1990, 1991; Damasio, 1994; Bechara et al., 1996, 1997). Moreover, using event-related analysis we found a dissociation between activity in left ventromedial prefrontal cortex during generation of SCR responses and activity in right ventromedial prefrontal cortex, reflecting feedback representation of evoked arousal. Despite this apparent dissociation, conjunction analysis of early and late activity indicated that ventromedial prefrontal activity is active bilaterally during both generation and representation of SCR events. These findings are consistent with the importance of this region in providing "somatic markers" that influence social and emotional behavior. The somatic marker hypothesis is derived from observations in patients with circumscribed deficits in social/emotional behavior and decision-making as a consequence of ventromedial prefrontal cortex lesions. Compared with normal control subjects, patients with ventromedial prefrontal cortical lesion perform badly on a gambling task, and unlike normal controls, fail to show anticipatory arousal before making a bad decision (i.e., one associated with a high probability of large monetary loss) (Bechara et al., 1996). Anticipatory arousal in 


\begin{tabular}{|c|c|c|c|c|c|}
\hline Brain region & Brodmann area & Side & Number of voxels & $Z$-score & Talairach coordinates $^{a}$ \\
\hline \multicolumn{6}{|c|}{ A, Activity preceding peaks of SCR events } \\
\hline Cerebellum & & $\mathrm{L}$ & 56 & $4.55^{b}$ & $-32-48-38$ \\
\hline Lingual gyrus & 18 & $\mathrm{~L}$ & 87 & 4.31 & $-14-80-4$ \\
\hline Cerebellum & & $\mathrm{R}$ & 34 & 4.08 & $24-52-38$ \\
\hline Medial prefrontal lobe & 10 & $\mathrm{~L}$ & 27 & 4.01 & -125010 \\
\hline Fusiform gyrus & 19 & $\mathrm{R}$ & 26 & 3.96 & $38-840$ \\
\hline \multicolumn{6}{|c|}{ B, Activity subsequent to peaks of SCR events } \\
\hline Medial prefrontal lobe & 10 & $\mathrm{R}$ & 156 & $4.63^{b}$ & $1052-10$ \\
\hline \multicolumn{6}{|c|}{$\begin{array}{l}\text { C, Common activity preceding and following } \\
\text { SCR events }\end{array}$} \\
\hline Lingual gyrus & 18 & $\mathrm{~L}$ & 268 & $4.92^{b}$ & $-16-80-8$ \\
\hline Medial prefrontal lobe & 10 & $\mathrm{~L}$ & 484 & $4.83^{b}$ & -14462 \\
\hline Posterior cingulate & 31 & $\mathrm{R}$ & 43 & 4.55 & $6-6218$ \\
\hline Medial prefrontal cortex & 10 & $\mathrm{R}$ & 110 & 4.51 & $654-14$ \\
\hline Orbitofrontal cortex & $11 / 47$ & $\mathrm{R}$ & 88 & 4.47 & $2824-22$ \\
\hline Fusiform gyrus & 18 & $\mathrm{R}$ & 83 & 4.32 & $36-90-4$ \\
\hline Lingual gyrus & 18 & $\mathrm{R}$ & 61 & 4.20 & $36-90-4$ \\
\hline Cerebellum & & $\mathrm{R}$ & 36 & 4.06 & $28-50-46$ \\
\hline
\end{tabular}

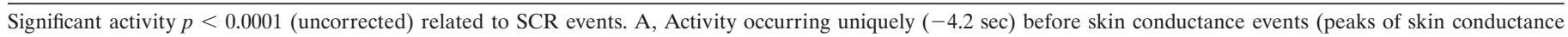

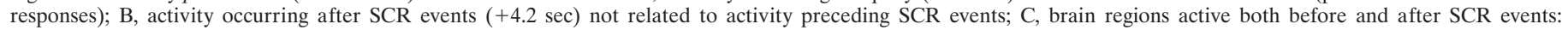
conjunction analysis of A and B. L, Left; R, right.

${ }^{a}$ Coordinates are given for the stereotactic space of Talairach and Tournoux (1988).

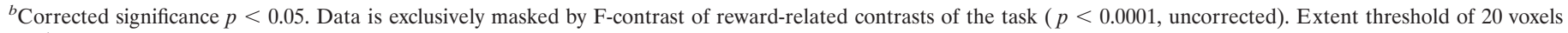
used.

controls, indexed by SCR, develops over the course of the gambling task and actually precedes explicit knowledge about the riskiness of particular decisions (Bechara et al., 1997). Because patients with ventromedial prefrontal lesions fail to show these anticipatory SCRs and make bad decisions, the somatic marker hypothesis proposes that feedback of somatic arousal directly influences motivational decision making and requires the integrity of ventromedial prefrontal cortex (Damasio et al., 1991; Damasio, 1994). Our observation of robust activity in bilateral medial prefrontal area 10 , directly related to somatic arousal (SCR events), suggests that somatic markers are represented within this region. However, because of technical limitations (susceptibility artifact), we cannot assess the extent to which adjacent regions in medial orbitofrontal cortex contribute to this somatic representation.

In the covariance and event-related analyses, SCR-related activity was not confined to prefrontal regions but was also observed in cerebellum, parietal, and occipitotemporal cortices. The cerebellum has been proposed as a component of a central autonomic network (Spyer, 1999). Autonomic pathways through the cerebellum are implicated in the representation of cardiovascular responses (Bradley et al., 1991; Critchley et al., 2000), conditioning (Gherlarducci et al., 1996), and autonomic components of emotional behavior (Martner, 1975). It is of note that these cerebellar activations occurred in the region of the dentate nucleus, lateral to the cerebellar vermis, which is more consistently implicated in autonomic control.

Surprisingly, we also observed SCR-related activity, in both the covariance analysis and event-related study, in extrastriate visual areas (lingual and fusiform gyri). Although we controlled for task-specific effects in our analyses, this activity is likely to represent modulation by arousal of areas involved in processing the visual stimuli that carry information about reinforcement. Extrastriate visual cortices (in particular the region of the lingual gyrus identified in our study) increase in activity when subjects process visual cues in association with states of high arousal, indexed by SCR (Lane et al., 1999). These data parallel the reported modulation of early visual cortices by attention (Chawla et al., 1999; Kastner et al., 1999). We propose that this modulation of early visual processing by arousal represents an adaptive mechanism for facilitating processing of motivationally relevant sensory information. A similar perspective may account for the observation that activity in the inferior parietal lobe (BA 40), covarying with SCR, represents a correspondence between arousal and attention.

The right inferior parietal lobe is implicated in directing attention, e.g., toward visual stimuli (Fink et al., 1996; Kastner et al., 1999), and arousing emotive stimuli (Peyron et al., 1999). Interestingly, lesions to right inferior parietal lobe also impair generation of SCR (Tranel and Damasio, 1994), and SCR is used as a behavioral measure of attention (Bouscein, 1992). However, lateral parietal activity was not apparent in the event-related analyses of our data, but lingual and fusiform gyri showed eventrelated activity both before and after the SCR events, consistent with independent modulation of extrastriate visual cortices by attention and peripheral arousal.

Although we observed activity in prefrontal and insula regions during SCR arousal, we did not observe much SCR-related activity in the anatomically and functionally connected areas of anterior cingulate cortex and amygdala (Carmichael and Price, 1996). Anterior cingulate activity is associated with physical and cognitive effort (Paus et al., 1998), sympathetic cardiovascular arousal (Critchley et al., 2000), and subjective emotional experience (Lane et al., 1997), and mid-cingulate activity has been reported previously in association with SCR (Fredrikson et al., 1998). This lack of anterior cingulate activity may reflect the transient nature of SCR events in contrast to sustained arousal evoked in other studies, or alternatively may represent anatomical 
A)
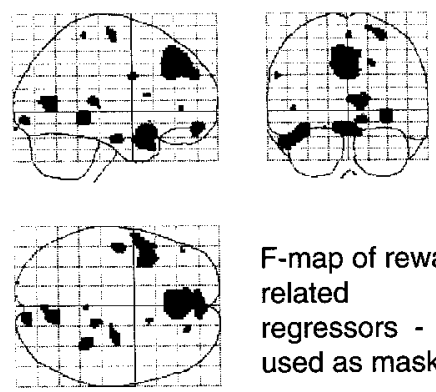

\section{F-map of reward-} related

regressors used as mask

B)
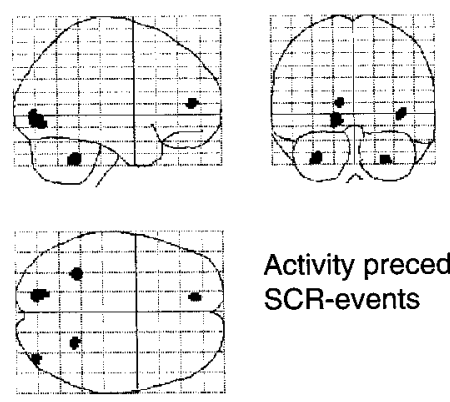

\section{Activity preceding} SCR-events

C)
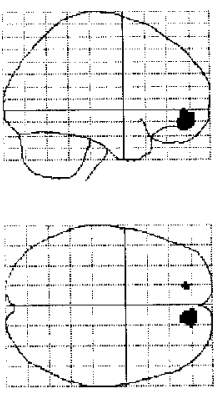

\section{Activity following} SCR-events

D)
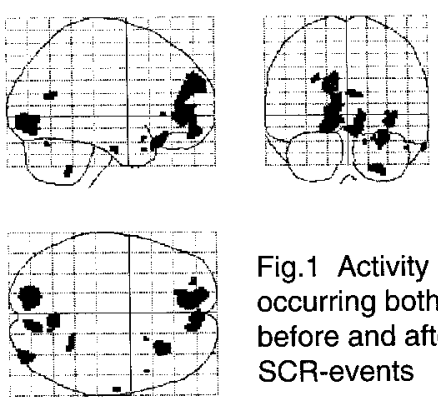

Fig.1 Activity occurring both before and after SCR-events

Figure 6. SPMs of contrasts of event-related analyses. $A$, SPM of the F-contrast of three reward-related regressors of the task representing the height of reward bar change in reward bar height and their interaction. The Figure depicts significant ( $p<0.0001$, uncorrected) regional activity (shaded area) attributable to variance in these regressors. This contrast was used as an exclusive mask for data reported in event-related analyses. A similar mask was used for data reported in the covariance analysis with the threshold at $p<0.0001$, uncorrected. The data are presented on a "glass brain." $B$, SPM of significant activity $(p<0.0001$, uncorrected) preceding SCR events (no mask present) corresponding to activity modeled with a hemodynamic response function occurring $4.2 \mathrm{sec}$ before SCR event). $C$, SPM of significant activity ( $p<0.0001$, uncorrected) after SCR events (no mask present) corresponding to activity modeled with an HRF occurring $4.2 \mathrm{sec}$ after SCR event and orthogonalized to the model used in $B . D$, SPM of significant activity ( $p<0.0001$, uncorrected) common to both generation and afferent representation of SCR, i.e., identified in a conjunction analysis of early and late SCR event-related activity. Figure shows unmasked SPM.
A

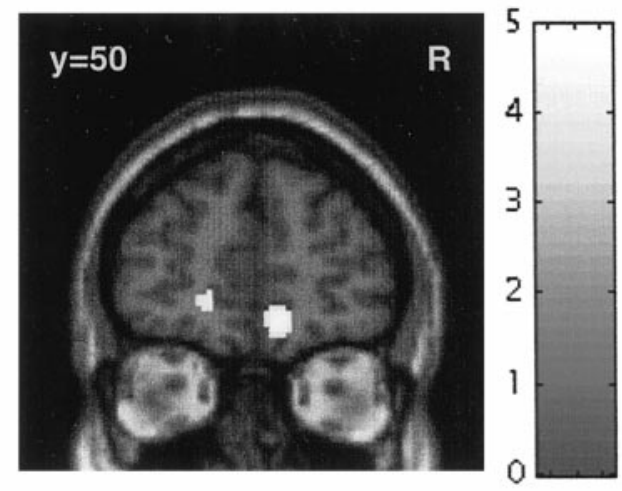

B
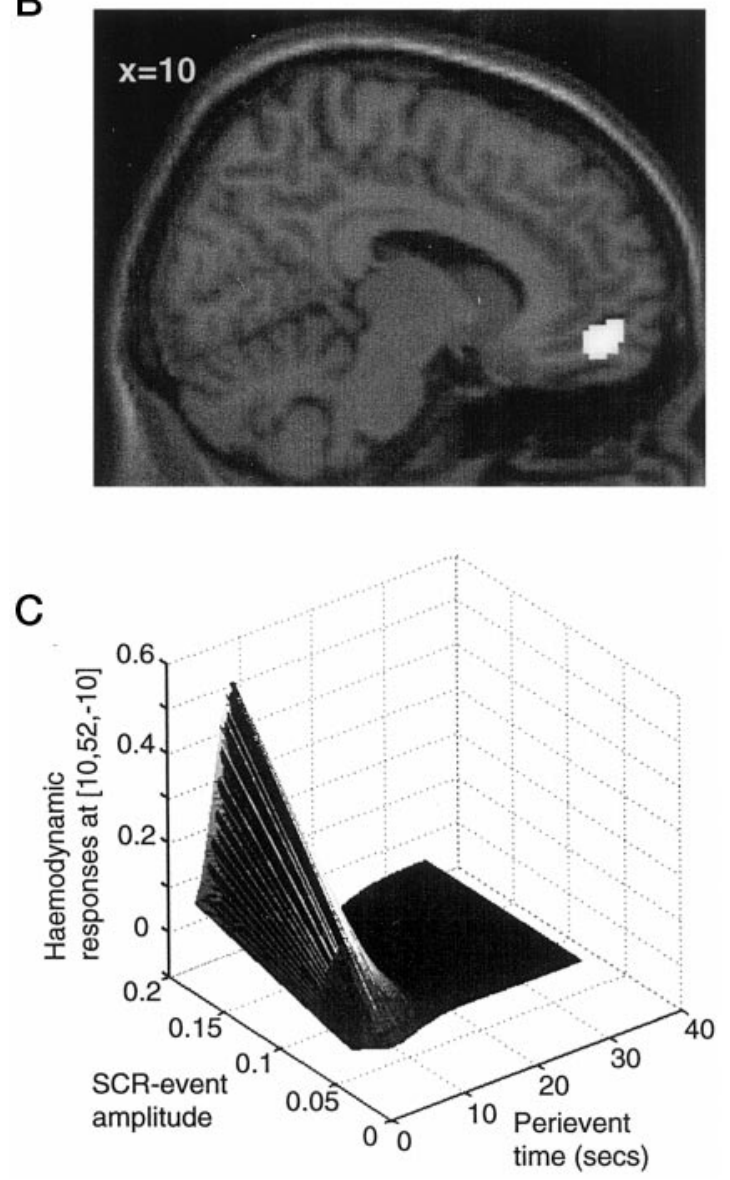

Figure 7. Ventromedial prefrontal activity consistent with feedback representation of SCR events. A, Activity depicted on a coronal section (52 $\mathrm{mm}$ anterior to the anterior commissure in standard space) of a single subject's brain for the purpose of presentation. The $Z$-score of the activity is given in the adjacent gray scale. $B$, Activity depicted on a parasagittal section of the right hemisphere ( $8 \mathrm{~mm}$ lateral in standard space). $C$, Parametric modulation of hemodynamic response in right ventromedial prefrontal cortex by amplitude of discrete SCR events in one subject. This effect was observed in five of the six subjects.

dissociation of central autonomic centers involved in the regulation of cardiovascular responses (conveyed centrally by the vagus nerve) from those representing electrodermal activity (conveyed by somatic afferents to the spinal cord).

The central representation of changes in somatic arousal is proposed to influence motivational behavior and may require the 
integrity of ventromedial prefrontal cortex. Our study examined regional brain activity associated with the SCR during rewardrelated behavior. In a covariance analysis, activity in right orbitofrontal cortex, right insula, bilateral medial frontal pole, cerebellum, and right inferior parietal lobe was associated with representation of SCR. Using event-related analyses, it was possible to dissociate activity in left medial prefrontal pole and cerebellum, related to generation of SCR events, from activity in right medial prefrontal lobe, related to afferent representation of SCR events. However, conjunction analysis demonstrated significant activity, related to both generation and representation of SCR events, in bilateral medial prefrontal and extrastriate cortices and right orbitofrontal cortex and cerebellum. Thus, we describe a set of discrete cortical brain regions in which somatic arousal (indexed by SCR) is represented during a reward-related decision-making task. These findings indicate that cognitive and emotional aspects of behavior are integrated with information regarding peripheral autonomic states of arousal, particularly in ventromedial and orbital frontal cortex.

\section{REFERENCES}

Bechara A, Tranel D, Damasio H, Adolphs R, Rockland C, Damasio A (1995) Double dissociation of conditioning and declarative knowledge relative to the amygdala and hippocampus in humans. Science 267:1115-1118.

Bechara A, Tranel D, Damasio H, Damasio AR (1996) Failure to respond autonomically to anticipated future outcomes following damage to prefrontal cortex. Cereb Cortex 6:215-225.

Bechara A, Damasio H, Tranel D, Damasio AR (1997) Deciding advantageously before knowing the advantageous strategy. Science 275:1293-1295.

Bechara A, Damasio H, Damasio AR, Lee GP (1999) Differential contributions of the human amygdala and ventromedial prefrontal cortex to decision making. J Neurosci 19:5473-5481.

Bouscein W (1992) Electrodermal activity. New York: Plenum.

Bradley DJ, Ghelarducci B, Spyer KM (1991) The role of the posterior cerebellar vermis in cardiovascular control. Neurosci Res 12:45-56.

Buchel C, Morris J, Dolan R, Friston K (1998) Brain systems mediating aversive conditioning: an event-related fMRI study. Neuron 20:947-957.

Cahill L (1997) The neurobiology of emotionally influenced memory. Implications for understanding traumatic memory. Ann NY Acad Sci 821:238-246.

Carmichael ST, Price JL (1996) Connectional networks within the orbital and medial prefrontal cortex of macaque monkeys. J Comp Neurol 371:179-207.

Cechetto DF, Saper CB (1987) Evidence for a viscerotopic sensory representation in the cortex and thalamus in the rat. J Comp Neurol 262:27-45.

Chawla D, Rees G, Friston KJ (1999) The physiological basis of attentional modulation in extrastriate visual areas. Nat Neurosci 2:671-676.

Critchley HD, Rolls ET (1996) Hunger and satiety modify the responses of olfactory and visual neurons in the primate orbitofrontal cortex. J Neurophysiol 75:1673-1686.

Critchley HD, Corfield DR, Chandler MP, Mathias CJ, Dolan RJ (2000) Cerebral correlates of autonomic cardiovascular arousal: a functional neuroimaging investigation. J Physiol (Lond) 523:259-270.

Damasio AR (1994) Descartes' error. New York: Putnam.

Damasio AR, Tranel D, Damasio HC (1990) Individual with sociopathic behaviour caused by frontal damage fail to respond autonomically to social stimuli. Behav Brain Res 41:81-94.
Damasio AR, Tranel D, Damasio HC (1991) Somatic markers and the guidance of behavior: theory and preliminary testing. In: Frontal lobe function and dysfunction (Levin HS, Eisenberg HM, Benton LB, eds), pp 217-229. New York: Oxford UP.

Duvernoy HM (1991) The human brain: surface three dimensional sectional anatomy and MRI. New York: Springer.

Fink GR, Halligan PW, Marshall JC, Frith CD, Frackowiak RS, Dolan RJ (1996) Where in the brain does visual attention select the forest and the trees? Nature 382:626-628.

Fredrikson M, Furmark T, Olsson MT, Fischer H, Andersson J, Langstrom B (1998) Functional neuroanatomical correlates of electrodermal activity: a positron emission tomographic study. Psychophysiology 35:179-185.

Friston K, Ashburner J, Frith CD, Poline J-B, Heather JD, Frackowiak RSJ (1995a) Spatial registration and normalization of images. Hum Brain Mapp 2:165-189.

Friston K, Holmes AP, Worsley K, Poline J-B, Frith C, Frackowiak RSJ (1995b) Statistical parametric maps in functional imaging: a general linear approach. Hum Brain Mapp 2:189-210.

Friston KJ, Holmes AP, Poline J-B, Grasby PJ, Williams SC, Frackowiak RS, Turner R (1995c) Analysis of fMRI time-series revisited. NeuroImage 1:45-53.

Ghelarducci B, Salamone D, Simoni A, Sebastiani L (1996) Effects of early cerebellar removal on the classically conditioned bradycardia of adult rabbits. Exp Brain Res 111:417-423.

James W (1894) Physical basis of emotion. Psychol Rev 1:516-529. Reprinted in: Psychol Rev 101:205-210, 1994.

Kastner S, Pinsk MA, De Weerd P, Desimone R, Ungerleider LG (1999) Increased activity in human visual cortex during directed attention in the absence of visual stimulation. Neuron 22:751-761.

Lane RD, Fink GR, Chau PM, Dolan RJ (1997) Neural activation during selective attention to subjective emotional responses. NeuroReport 8:3969-3972.

Lane RD, Chua PM, Dolan RJ (1999) Common effects of emotional valence arousal and attention on neural activation during visual processing of pictures. Neuropsychologia 37:989-997.

Mangina CA, Beuzeron-Mangina JH (1996) Direct electrical stimulation of specific human brain structures and bilateral electrodermal activity. Int J Psychophysiol 22:1-8.

Martner J (1975) Cerebellar influences on autonomic mechanisms. Acta Physiol Scand [Suppl] 425:1-43.

Oppenheimer SM, Gelb A, Girvin JP, Hachinski VC (1992) Cardiovascular effects of human insular cortex stimulation. Neurology 42:1727-1732.

Oscar-Berman M, Gade A (1979) Electrodermal measures of arousal in humans with cortical or subcortical brain damage. In: The orientating reflex in humans (Kimmel H, ed), pp 665-676. Hillsdale, NJ: Erlbaum.

Paus T, Koski L, Caramanos Z, Westbury C (1998) Regional differences in the effects of task difficulty and motor output on blood flow response in the human anterior cingulate cortex: a review of 107 PET activation studies. NeuroReport 9:R37-R47.

Peyron R, Garcia-Larrea L, Gregoire MC, Costes N, Convers P, Lavenne F, Mauguiere F, Michel D, Laurent B (1999) Haemodynamic brain responses to acute pain in humans: sensory and attentional networks. Brain 122:1765-1780.

Rolls ET, Critchley HD, Mason R, Wakeman EA (1996) Orbitofrontal cortex neurons: role in olfactory and visual association learning. J Neurophysiol 75:1970-1981.

Spyer KM (1999) Central nervous control of the cardiovascular system. In: Autonomic failure: a textbook of clinical disorders of the autonomic nervous system, Ed 4 (Mathias CJ, Bannister R, eds), pp 45-55. Oxford, UK: Oxford UP.

Talairach J, Tournoux P (1988) Co-planar stereotaxic atlas of the human brain. Stuttgart: Theime.

Tranel D, Damasio H (1989) Intact electrodermal skin conductance responses after bilateral amygdala damage. Neuropsychologia 27:381-390.

Tranel D, Damasio H (1994) Neuroanatomical correlates of electrodermal skin conductance responses, Psychophysiology 31:427-438.

Zoccolotti P, Scabini D, Violani C (1982) Electrodermal responses in patients with unilateral brain damage. J Clin Neuropsychol 4:143-150. 